\title{
Experiment of Polarization Forces in Scanning Electrostatic Force Microscopy for Measuring Surface Profile of Dielectric
}

\author{
Gaofa $\mathrm{HE}^{1,2}$, Zhigang $\mathrm{JIA}^{2}$,So ITO ${ }^{2}$, Yuki SHIMIZU², Wei GAO ${ }^{2}$ \\ ${ }^{1}$ College of Mechanical and Power Engineering, Chongqing University of Science and Technology, China, \\ hegaofa@nano.mech.tohoku.ac.jp \\ ${ }^{2}$ Nano-metrology and Control Laboratory, Department of Nanomechanics, Tohoku University, Sendai, Japan
}

\begin{abstract}
:
For measuring the surface profile of many micro-optical components with complicated shapes, which are made of non-conductive material, the electrostatic force microscopy (EFM) was recommended. The relationship between the polarization force and the tip-to-sample distance was analyzed based on dielectric polarization theory. The prototype of the scanning electrostatic force microscopy was built. The force curves of different samples with different materials and surface shapes were detected by the EFM prototype. Both theoretical analysis and the experimental results demonstrated that the EFM system can be used to measure the surface profile of non-conductor.
\end{abstract}

Keywords: Electrostatic force microscopy (EFM), Profile measurement, Dielectric, Polarization

\section{Introduction}

For measuring the three-dimensional (3D) microstructures surfaces profile, scanning probe microscopy (SPM) is one of the most powerful tools ${ }^{[1,2]}$. The representative SPM are scanning tunneling microscopy (STM) and atomic force microscopy (AFM). However, the STM can only be used for measuring the surface of conductor ${ }^{[3]}$. When the AFM works at the contact mode and the tapping mode, the large contact stress will damage the sample surface because of the sharp tip of the scanning probe ${ }^{[4]}$. Although the contact force can be reduced and the damage of the sample surface can be avoided by the use of the noncontact mode AFM (NC-AFM), it is necessary to maintain a very small tip to sample separation, less than $5 \mathrm{~nm}$, so that the weak interactions of atomic force between the tip and the sample, which is only effective in a short tip to sample separation, can be detected ${ }^{[5]}$. This makes the probe tip of the NC-AFM scans over the sample surface with a slow speed for avoiding the tip from colliding with the surface. For conventional SPM, both STM and AFM, it is difficult to measure surface profiles of microstructures with steep local slopes and large amplitudes, such as diffraction gratings, micro-lens arrays and micro-prism sheets.

Recently, the electrostatic force microscopy (EFM) was also proposed for the measurement of the microstructure surface profile ${ }^{[6]}$. This EFM system, which can scan over the surface with a large tip to sample separation of up to several hundreds of nanometers by utilizing the long range electrostatic force, was build to measure the surface profiles for these micro-optical components. In that paper, it only discussed the measurement method about the conductive surface. In this paper, the EFM system for non-conducting surface profile measurement was proposed. The measuring principle is discussed in theory and a prototype EFM measurement system was built for demonstrating the feasibility of the proposed method.

\section{Measurement Principle}

A conducting probe tip is oscillated with small amplitude in the order of $10 \mathrm{~nm}$ by a tuning fork quartz crystal resonator at their resonance frequency of the oscillation system. The sample is attached on the back electrode as shown in Fig. 1 and the distance between the probe tip and the sample surface is denoted by $d$. The oscillation amplitude is set to be smaller than $d$ so that the tip will not contact with the surface. When a bias voltage $V_{\mathrm{dc}}$ is applied on the probe tip and the electrode, the sample which is set in the electrical field will be polarized and the polarization charge will be produced on the sample surface ${ }^{[7]}$. The electrostatic force $F$ between the probe tip and the sample surface can be expressed. The value of electrostatic force depends on $d$ and voltage $\left(V_{\mathrm{dc}}\right)$ between the tip and sample surface. Because the electrostatic force acts as an external force to the oscillation system which consisting of the resonator and the probe tip, the effective resonance frequency of the system will be changed by the electrostatic force. Then the relationship between the frequency shift of the oscillation system $\Delta f$ and the tip-sample distance $d$ can be obtained. If the $\Delta f$ is detected, $d$ can be gotten. So, according to the frequency shifting value the profile of the sample surface can be acquired.

When the dielectric is put in an electrical field, polarization charges will emerge on the sample surface. So, the electrostatic force will be loaded on the probe tip, which can be calculated according to polarization theory. As shown in Fig. 1, when a bias voltage is loaded on the back electrode, the positive charges will be produced on the surface of the electrode. Then, on the upper surface and the under surface of the sample positive charges and negative charges will emerge, respectively, and the image charges also will be produced on the tip of probe. 
Position of probe in $\mathrm{Z}$

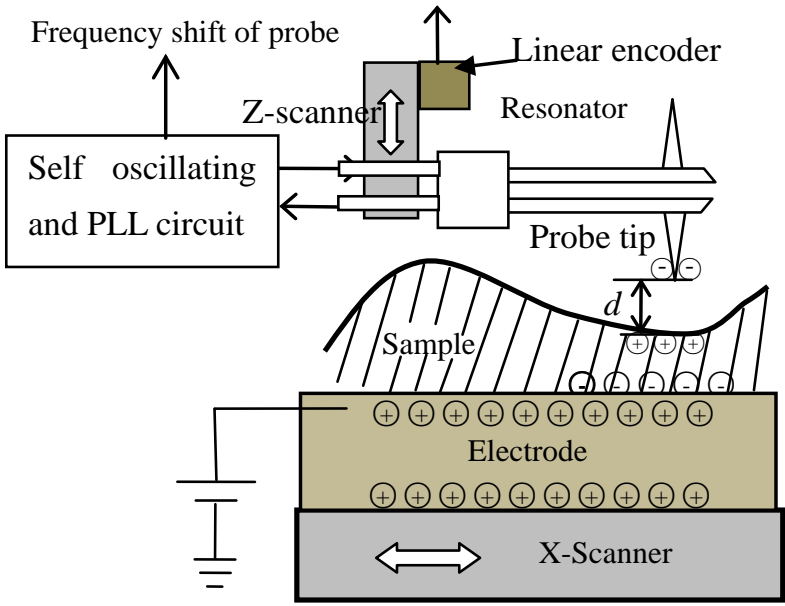

Figure1: Principle of EFM

The electric susceptibility of the sample, the dielectric constant of vacuum and the permittivity of the sample is denoted by $\chi, \varepsilon_{0}$, and $\varepsilon_{\mathrm{r}}$ respectively. $U_{\mathrm{bt}}$ is the potential difference between the back electrode and the probe tip.

The force between the tip and the sample can be calculated as:

$$
F=\frac{\varepsilon_{0}}{2}\left[1-\frac{h}{\varepsilon_{r}(h+d)}\right]^{2} U_{b t}^{2} \frac{A}{d^{2}},
$$

where $A$ is the area of the half sphere of probe apex, $d$ is the tip-sample distance and $h$ is the thickness of the sample.

The resonance frequency $f_{0}$ of the resonator will be changed by the force shown in Eq. (1). The frequency shift $\Delta f$ can be written as ${ }^{[6]}$ :

$$
\Delta F=\frac{f_{0}}{2 k} F^{\prime},
$$

where, $k$ is the spring constant of the oscillation system, and the $F^{\prime}$, which means the electrostatic force gradient, is defined as: $F^{\prime}=\partial F / \partial d$.

If the thickness of the sample $h$ is far greater than the distance $d$, equation (1) can be written as:

$$
F=\frac{\varepsilon_{0}}{2}\left(1-\frac{1}{\varepsilon_{r}}\right)^{2} U_{b t}^{2} \frac{A}{d^{2}} .
$$

Thus,

$$
F^{\prime}=\partial F / \partial d=-\varepsilon_{0}\left(1-\frac{1}{\varepsilon_{r}}\right)^{2} U_{b t}^{2} \frac{A}{d^{3}} .
$$

Combining the Eq. (2) and (4), the frequency shift can be expressed as:

$$
\Delta f=-\frac{\varepsilon_{0}}{2}\left(1-\frac{1}{\varepsilon_{r}}\right)^{2} U_{b t}^{2} \frac{A}{d^{3}} \frac{f_{0}}{k} .
$$

As can be seen in Eq. (5), the frequency shift value $\Delta f$ is a function of $d$, which is related to the surface profile.

\section{Prototype EFM Measurement System}

With the similar principle of the frequency modulation (FM) AFM, the prototype of a scanning electrostatic force microscopy for profile measurement was built. The prototype system will be used for measuring the frequency shift with the distance between the tip and the sample surface. According to the frequency shift of the tuning fork resonator, the electrostatic force between tip and sample can be calculated and then the surface profile can be imaged. Figure2 shows a schematic of the prototype EFM measurement system. The EFM probe unit was mounted on a $\mathrm{Z}$ scanner which was driven by piezoelectric actuators. The $\mathrm{Z}$ scanner employed a linear encoder for accurate measurement of the probe displacement along the $\mathrm{Z}$ direction over a range of $50 \mu \mathrm{m}$ with a nonlinear error of less than $10 \mathrm{~nm}$. The sample and the back electrode were mounted on an XY scanner which was also driven by piezoelectric actuators. The commercial XY scanner was feedback controlled by using capacitive displacement sensors over a travel stroke of $100 \mu \mathrm{m}$ with a nonlinear error of approximately $40 \mathrm{~nm}$ in each of the $\mathrm{X}$ and $\mathrm{Y}$ directions ${ }^{[6]}$.

Fig. 3 shows the image of the probe unit. Two pieces of tungsten probe tip which were made by electrochemically polishing method were glued on a tuning fork quartz crystal resonator with conductive epoxy. One of the probe tips is utilized as a counterweight for balance, and the other tip is employed for detecting the electrostatic force. The probe tips were oscillated with the resonator at the fundamental resonance frequency of the resonator by using a self oscillating circuit. With regard to the variation of the resonance frequency, the frequency shift signal was detected by using the phase locked loop (PLL) circuit and

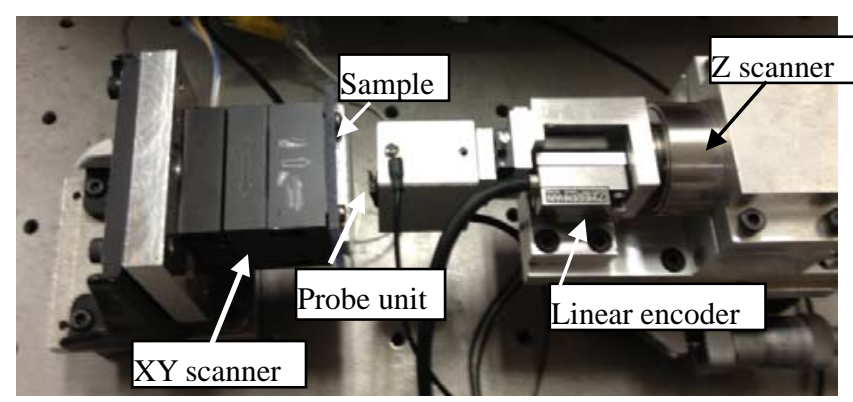

Figure 2: Schematic of the prototype EFM

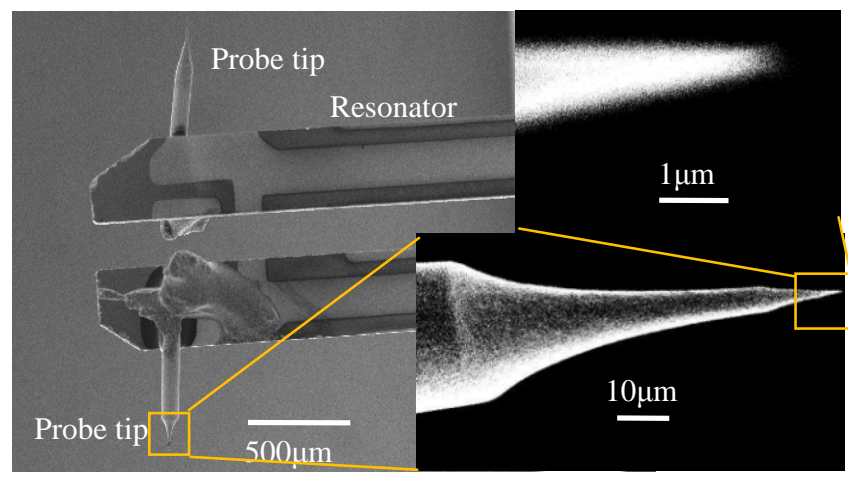

Figure 3: SEM image of probe tip 
then the signal is fed into the computer for profile exploring and also sent to the proportional integral (PI) controller for controlling the probe tip to scan the sample surface with constant force mode.

\section{Experiment}

\subsection{Experiment on Different Material}

Form the Eq. (5), it can be seen that the frequency shift value $\Delta f$ is also a function of the permittivity of the sample $\varepsilon_{\mathrm{r}}$. That means the frequency shift is affected by material. To confirm the feasibility of using the EFM system to measure surface profile of the dielectric, the frequency of the resonator shifting changes with different distances between the probe tip and sample surface on different dielectrics were tested. Firstly, a glass plate was employed as the sample and an aluminum alloy plate was used as the back electrode. The bias voltage applied between the probe tip and the back electrode was set to be $100 \mathrm{~V}$. The probe was oscillated at the fundamental frequency of resonator which was about $26546 \mathrm{~Hz}$ in this experiment. The quality factor of oscillation system was 2832. The sample was kept stationary and the probe tip was moved by the $\mathrm{Z}$ scanner to approach to the sample surface for investigating the relationship between the frequency shift and the tip to sample distance. The result is shown in Fig. 4. The vertical axis shows the corresponding frequency shifts. The bottom horizontal axis shows the outputs of the linear encoder of the $\mathrm{Z}$ scanner, which represents the relative displacement of the $\mathrm{Z}$ scanner. The results can be well fitted by an inverse cubic function by using the least squares method, which reflects the relationship shown in Eq. (5). The inverse cubic fitting curve can be used to estimate the absolute tip to sample distance based on Eq. (5). In Fig. 4, the upper horizontal axis is the estimated absolute tip to sample distance fitted by least squares method.

Then, with the same procedure, a silicon slice and a PVC film were taken as the sample and tested respectively. Figure 5 and Fig. 6 are the test results. The three figures show that these materials can be test by the EFM system; and the signal when the sample is PVC is weakest because of its least $\varepsilon_{\mathrm{r}}$.

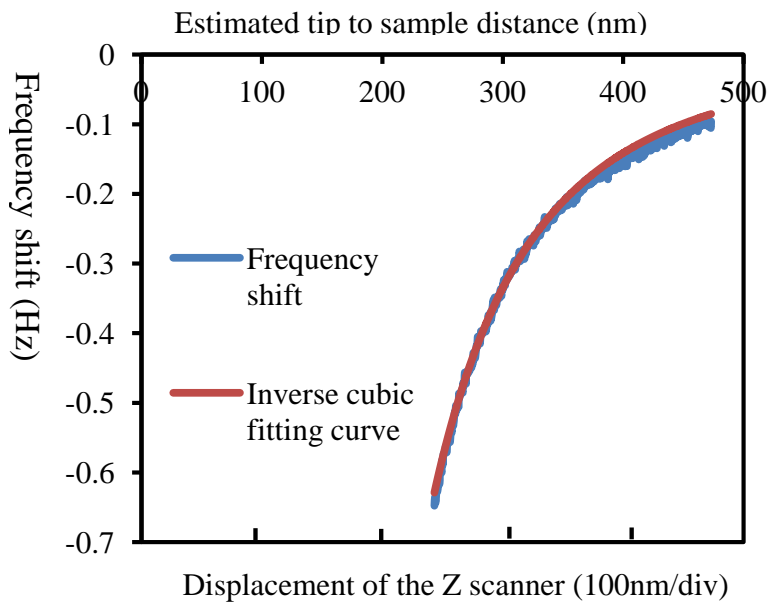

Figure 4: Relationship between $d$ and $\Delta f$ on glass plate

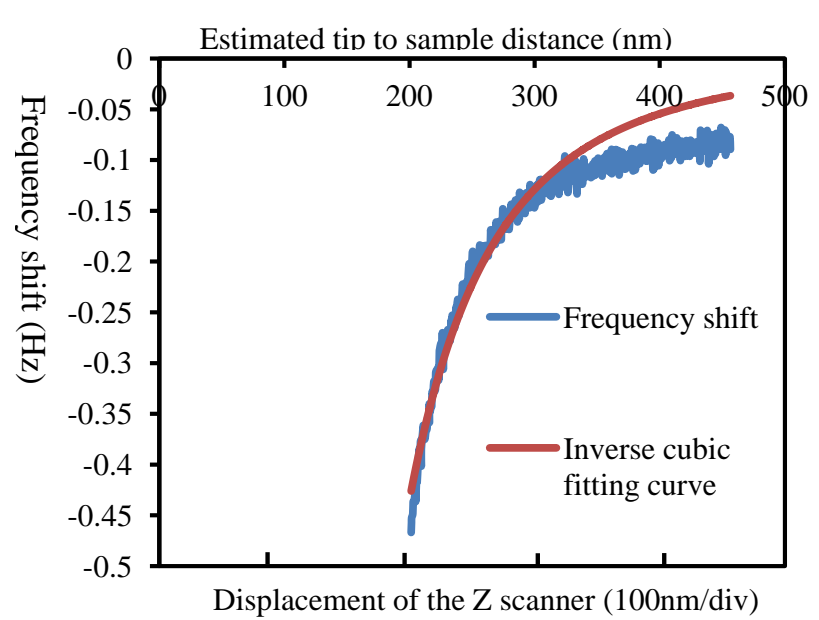

Figure 5: Relationship between $d$ and $\Delta f$ on silicon slice

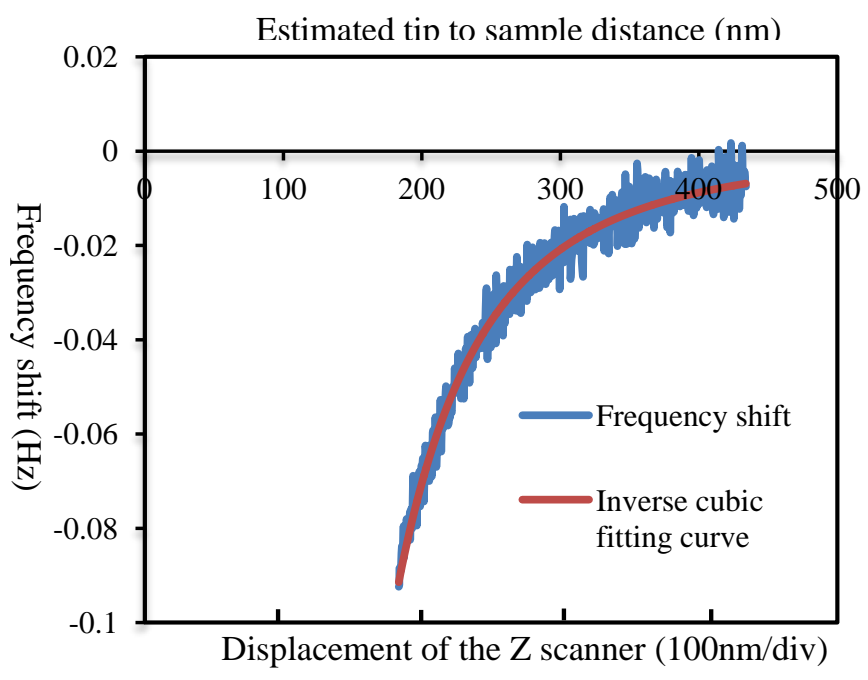

Figure 6: Relationship between $d$ and $\Delta f$ on PVC film

\subsection{Experiment on Different Position}

According to the polarization theory, the topology of the sample surface will effect on the electrostatics force. For researching the effect by the topology of the surface, a diffraction grating with $25 \mu \mathrm{m}$-high ridges was employed as the testing sample. Figure 7 shows the topography of the sample surface. The right image is the section form of the ridges.

Along $\mathrm{X}$ direction within $50 \mu \mathrm{m}$-length, the force curves at 50 points were tested by X-scanner moved with $1 \mu \mathrm{m}$ step, in order to insure that the top and the valley points on the ridge can be tested (shown as in Fig. 8). Force curve at each point was obtained. Figure 9 shows three of them.

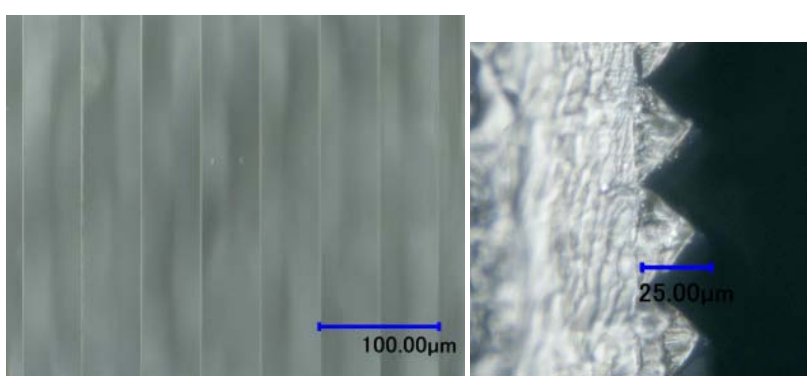

Figure 7: Image of the sample with $25 \mu \mathrm{m}$ - high ridge 


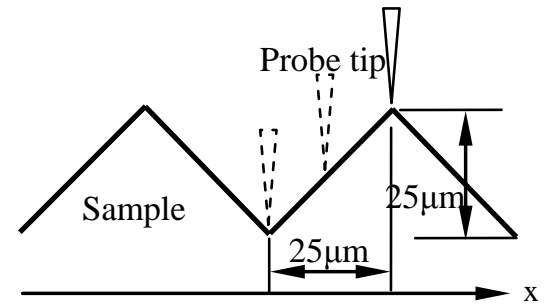

Figure 8: Testing method of force curve on grating

Estimated tip to sample distance (nm)

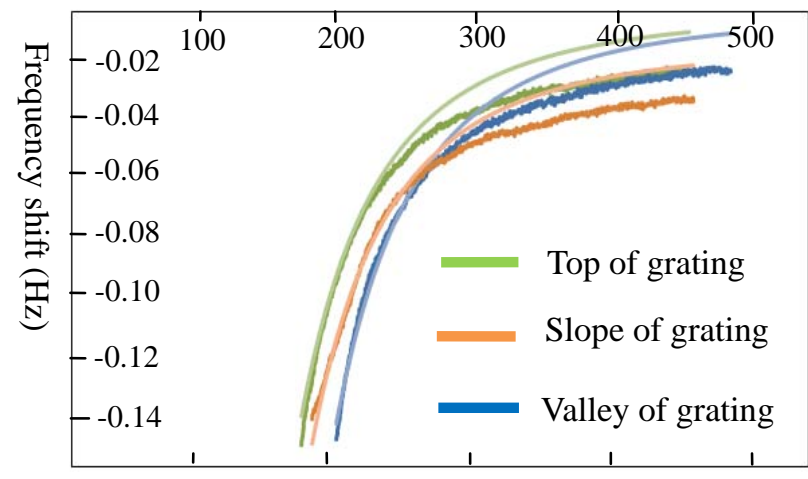

Displacement of the Z scanner (100nm/div)

Figure 9: Relationship between $d$ and $\Delta f$ on grating

They are the force curves on the top, the slope and the valley of the ridge respectively. In Fig. 9, the smooth curves are the fitted curves and the crude curves are the tested curves.

\section{Conclusion and Future Works}

Some of optical components, such as diffraction gratings, micro-lens arrays and micro-prism sheets etc, are made of non-conductive material. On the surface of these components, there are many complicated shapes with steep slopes and large amplitudes. The electrostatic force microscopy (EFM) was proposed to measure surface profiles of microstructure. For confirming the feasibility, according to the polarization theory, the electrostatic force between the conductive probe tip and the non-conductive sample was calculated. The result shows that the relationship between the electrostatic force and the distance from probe tip to sample surface is inverse square function.

A prototype of EFM has been developed for measuring the surface profile of non-conductor in noncontact condition.

Experiments have also been carried out by using the prototype EFM system to demonstrate the feasibility of the electrostatic force calculation by the analytic method. A glass plant, a silicon slice and a PVC film was employed as the sample respectively. The bias voltage applied between the probe tip and the back electrode was set to be $100 \mathrm{~V}$. The fundamental frequency and the quality factor of the probe unit were tested. The values of them are $26546 \mathrm{~Hz}$ and 2832, respectively. The samples were kept stationary and the probe tip was moved by the $\mathrm{Z}$ scanner to approach to the sample surface for investigating the relationship between the frequency shift and the tip-to-sample distance. The test result shows that the frequency shifts with the tip-sample distance changes as the analytic result in theory. Then, a diffraction grating with $25 \mu \mathrm{m}$ high ridge was employed as the sample to test the effect of the topography. All results indicate that the EFM system can be used to measure the microstructure surface profile of dielectric.

About the future works, firstly, the accurate relationship between the electrostatic force and the tip-sample distance should be investigated by using the other method. Then, according to the force curve, scanning measurement along $\mathrm{X}$ and $\mathrm{Y}$ direction will be done for imaging the surface profile.

\section{Acknowledgement}

This research is supported by Japan Society for the Promotion of Science (JSPS) and Osawa Scientific Studies Grants Foundation. The authors would like to acknowledge JSPS and Osawa Scientific Studies Grants Foundation. HE thanks the China Scholarship Council (CSC) for financial assistance during his visit to Tohoku University.

\section{References}

[1] Wei Gao, J. Aoki, B. F. Ju and S. Kiyono, 2007, Surface profile measurement of a sinusoidal grid using an atomic force microscope on a diamond turning machine, Precision Engineering, Vol.31, No.3 pp.304-309.

[2] Clemens Barth , Adam S. Foster , Claude R. Henry , and Alexander L. Shluger, 2011, Recent Trends in Surface Characterization and Chemistry with High-Resolution Scanning Force Methods, Advanced Materials, Vol.23, No.4, pp.477-501.

[3] Christopher E.D. Chidsey, Dominic N. Loiacono, Tycho Sleator, Sho Nakahara, 1988, STM study of the surface morphology of gold on mica, Surface Science, Vol.200, No.1, pp. 45-66.

[4] Ralf B, C González, Jens S, Pavel J, Rubén P and Angelika K., 2009, 'All-inclusive' imaging of the rutile TiO2(110) surface using NC-AFM, Nanotechnology, Vol.20, No.50, pp.1-7.

[5] Mikhail Dunaevskiy, Prokhor Alekseev, Paul Girard, 2012, Analysis of the lateral resolution of electrostatic force gradient microscopy, Journal of Applied Physics, Vol.112, No.6, pp.1-7.

[6] Wei Gao, Shigeaki Goto, Keiichiro Hosobuchi, 2012, A noncontact scanning electrostatic force microscope for surface profile measurement, CIRP Annals-Manufacturing Technology, Vol.61, No.1, pp.471-474.

[7] Jun Hu, XuDong Xiao, and Miquel Salmeron, 1995, Scanning polarization force microscopy: A technique for imaging liquids and weakly adsorbed layers, Appl. Phys. Lett., Vol 67, No.4, pp.476-479. 